\title{
Be caught napping: you're doing more than resting your eyes
}

\author{
Pierre Maquet, Philippe Peigneux, Steven Laureys and Carlyle Smith \\ Carlyle Smith is in the Department of Psychology, Trent University, Peterborough, Canada. The other authors \\ are at the Cyclotron Research Centre, University of Liège, Liège, Belgium
}

\begin{abstract}
Sleep is suggested to repair fatigue or to enhance memory consolidation. $A$ new paper shows that the beneficial effect of sleep is specific to the task and the brain regions enqaqed by it.
\end{abstract}

Ever felt burned out after several hours of intense intellectual work? Ever tried a short nap after lunch to improve your productivity? In this issue, Mednick et al. suggest that you should. Their data show that a nap restores performance after repetitive training on the same day on a perceptual learning task ${ }^{1}$.

What happens in your brain during sleep? According to one hypothesis, sleep is involved in restoring brain function after the wear and tear of the day's activities. Such use-dependent processes are intimately linked to the neuronal workload of the preceding waking period (Fig. 1b, top). Another hypothesis is that sleep is at least favorable, if not indispensable, for memory consolidation in the brain. These experience-dependent processes entail expansion of the behavioral repertoire after exposure to a new environment. In contrast to restoration, consolidation might go on long after the last training sessior has ended (Fig. 1b, bottom) ${ }^{2}$.

The texture discrimination task described by Kami et $a l^{3}$ has beer exceptionally useful for the behavioral study of these two sleep functions in humans. In this task, a target consisting of a horizontal or vertical array of three diagonal bars is displayed briefly against a background of horizontal bars, followed by a blank screen, then by a mask The interval between the target and the mask (interstimulus interval, or ISI) is variable, and the ISI needed to achieve $80 \%$ correct responses is taken as a measure of perceptual ability. This procedure has become a classic task to investigate the role of sleep in memory consolidation. After a single training session, performance on this task improves only after subjects have slept during the first night after training ${ }^{2}$ (ISI decreases by $30 \mathrm{ms,}$ Fig.1a). This slow learning process is sensitive to REM sleep deprivation ${ }^{4}$ and is optimal only when subjects have had both non-REM sleep (early in the night) and REM sleep (late in the night) ${ }^{5}$.

Now Mednick et al. ${ }^{l}$ show that subjects' performance deteriorates if they are trained on the task four times at regular intervals on the same day. The more they are trained, the worse their performance becomes (about $40 \mathrm{~ms}$ increase in ISI after the fourth session; Fig. 1 in ref. 1). This deterioration can be avoided if the subjects are allowed to nap for 30 to 60 minutes at the beginning of the afternoon, as expected from previous studies (for instance, ref. 6). Importantly, the authors went on to show that performance does not deteriorate if stimuli in the last training session are presented in the visual hemifield contralateral to the one used in the initial training sessions, that is, when the probed visual cortex is contralateral to the initially trained cortex. This observation leads to the important conclusion that the deterioration in brain function is task dependent and regionally specific. In other words, it is not a global effect of fatigue on the brain, but a specific effect on the brain regions involved in the task. A 60-minute nap is more efficient than a 30-minute nap to recover the initial level of performance, the main difference being more time spent in non-REM sleep during long naps. These data can be viewed as evidence for a restorative effect of sleep, especially non-REM sleep, on regional brain function. They echo other examples of regionally specific, use dependent sleep processes in which increased stimulation during -wakefulness is followed by a enhanced intensity of sleep only in the corresponding sensory cortex ${ }^{7,8}$. For instance, in humans, after prolonged vibratory stimulation of the hand, slow-wave activity (an EEG marker of the intensity of slow-wave sleep) is specifically increased over the contralateral somatosensory cortex during subsequent sleep ${ }^{7}$. These previous studies, however, did not demonstrate a regionally specific functional benefit of sleep.

Although the authors discuss their findings in terms of the preservation of information in the cerebral networks as steps in memory formation and consolidation, the behavioral data only suggest the restoration of an optimal brain function during sleep. At the cellular and molecular levels, the cerebral correlates of the effects reported by Mednick et al. ${ }^{l}$ are still a matter of speculation. The restorative mechanisms are still poorly understood and might involve, for instance, the elimination of toxic compounds (for example, the response to oxidative stress by glutathione ${ }^{9}$ ) and the reconstitution of energy stores (such as brain glycogen ${ }^{10}$ ). Likewise, the mechanisms of consolidation of memory traces during sleep are still unknown, although gene transcription and protein synthesis are probably involved ${ }^{11}$. What is known, however, is that neural activity enhances the local release of many compounds like NO, adenosine or cytokines (IL1 $\beta, \mathrm{TNF} \alpha$, growth factors, interferons $\alpha$ and $\gamma$, and many others $)^{12}$. The level of some of these molecules increases with sleep need, suggesting that they might signal that neurons have been working for an extended period of time ${ }^{12,13}$. These molecules are not only released in a usedependent manner but participate in a cascade of molecular events that eventually promote sleep, and especially 
non-REM sleep (Fig. 1 in ref. 12).

Finally, some authors suggest that several of these molecular compounds, especially growth factors, not only induce sleep but are also involved in synaptic plasticity ${ }^{12,14}$. However, their role remains to be established in the consolidation of memory traces during sleep. The view thus emerges that a task-dependent, regionally specific set of neural networks challenged by previous waking activity could be identified by the production and release of a number of molecules. These compounds would promote sleep and lead to a cascade of processes involved in cerebral restoration, brain plasticity or both. To gain a comprehensive understanding of these mechanisms, future research will have to characterize these processes more fully.

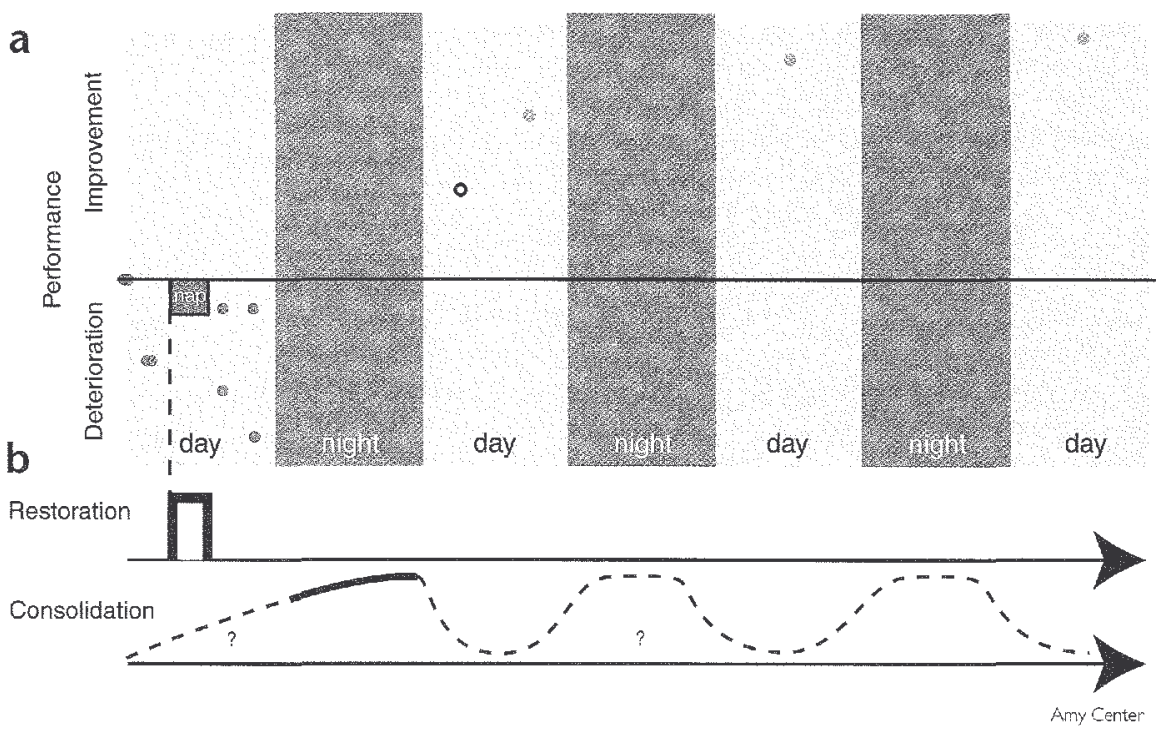

Fig. I. The role of sleep in memory, (a) The texture detection task is thought to rely on plastic changes in the primary visual cortex. With four sessions on the same day, performance worsens monotonically (red dots), unless the subjects can nap (purple square and dots). After a single training session, the performance improves after the first night's sleep. This improvement is significant if the subjects can sleep during the first part of the night (mainly non REM sleep, white dot) but is optimal only if they sleep the whole night (both non REM sleep and REM sleep, first blue dot). Performance still improves after the second and third nights (last two blue dots), (b) The hypothetical time course of restorative processes (top) and consolidation of memory traces (bottom). Restoration and memory processing are usually considered as two different sleep functions. At the cellular level, it is not known whether restoration and consolidation processes are distinct or have mechanisms in common.

\section{References}

[1] Mednick, S. et al. Nat. Neuros. 5, 677-681 (2002).

[2] Stickgold, R., James, L. \& Hobson, J. A. Nat. Neuros. 3, 1237-1238 (2000)

[3] Kami, A. \& Sagi, D. Proc. Natl. Acad. Sd. USA 88, 4966-4970(1991).

[4] Kami, A., Tarme, D., Rubenstein, B. S., Askenasy, J. J. \& Sagi, D. Science 265, 679- 682(1994).

[5] Gais, S., PIihal, W., Wagner, U. \& Born, J. Nat. Neuros. 3, 1335-1339 (2000).

[6] Bonnet, M. H. \& Aland, D. L. J. Sleep Res. 4, 71-77(1995).

[7] Kattler, H., Dijk, D. J. \& Borbely, A. A. J. Sleep Res. 3,159-164 (1994).

[8] Vyazovskiy, V., Borbely, A. A. \& Tobler, I. J. Sleep Res. 9, 367-371 (2000).

[9] Inoue, S., Honda, K. \& Komoda, Y. Behav. Brain Res. 69,91-96(1995).

[10] Benington, J. H. \& Heller, H. C. Prog. Neurobiol. 45, 347-360 (1995).

[11] Maquet, P. Science 294, 1048-1052 (2001).

[12] Krueger, J. M., Oba!, E J., Fang, J., Kubota, T. \& Taishi, P. Ann. NY Acad. Sd. 933, $211-221$ (2001).

[13] Strecker, R. E. et al. Behav. Brain Res. 115, 183-204 (2000).

[14] Brandt, J. A. et al. Brain Res. 898, 105-112 (2001). 